\title{
Reliability of the diagnosis of impairments in survey research in the field of chiropody
}

\author{
M. J. W. M. VAN GISBERGEN, J. DEKKER and W. ZUIJDERDUIN
}

\section{Accepted for publication: September 1992}

Keywords ICIDH, chiropody

\section{Summary}

Chiropody is a rather unknown paramedical profession in The Netherlands. A chiropodist treats foot and nail problems or postural deviations which may be corrected by means of remedial foot therapy. There is no history of scientific research on chiropody. A necessary first step is a survey on professional practice, diagnosis and treatment. A requirement for a survey study is a reliable registration form. In this study a registration form that draws on the conceptual framework of the ICIDH (International Classification of Impairments, Disabilities and Handicaps) was tested on inter-observer reliability. A chiropodist and a trainee examined 49 patients. Reliability was determined using two measures: the percentage of agreement and Cohen's Kappa. Findings of this study indicate that the reliability of the registration form for the assessment of the chiropody diagnosis is satisfactory.

\section{Introduction}

The need for research on Dutch paramedical professions such as chiropody has been recognized only recently. The need for information especially concerns professional practice, diagnosis and treatment by chiropodists. ${ }^{1}$ Chiropody is rather unknown in The Netherlands, whereas in the United Kingdom and the United States it is a quite well-known profession. A chiropodist treats mainly foot and nail problems or postural deviations which can be corrected by means of remedial foot therapy. A chiropodist can use various treatments, varying from temporary therapy such as wound treatment, bandaging and taping to chiropedic soles, nail braces or even prostheses. ${ }^{2}$ There are approximately 140 chiropodists in The Netherlands. Most of them (about 95\%) work in primary health care in private practice. Only a few chiropodists work in institutional care.

Chiropody does not have a tradition in The

Correspondence to: J. Dekker, Netherlands Institute for Primary Health Care, P.O. Box 1568, 3500 BN Utrecht, The Netherlands.
Netherlands. It developed 12 years ago out of several professions which were concerned with foot care. There is no history of scientific research on chiropody practice and few data on the practice of this profession are available. Therefore a study aimed at describing characteristics of patients, diagnosis by chiropodists and their interventions is regarded as a necessary first step in scientific research on chiropody. This kind of research provides knowledge to fill in the forementioned gaps, and further research, aimed at assessing the effects of certain applications, can be initiated based on the findings.

Because of the lack of scientific research on chiropody, it was first necessary to develop an instrument aimed at the assessment of diagnosis and treatment by chiropodists. The International Classification of Impairments, Disabilities and Handicaps ${ }^{3}$ (ICIDH) is considered an adequate system to classify diagnostic data concerning functional problems. In the ICIDH the consequences of disease are described in terms of impairments (consequences of disease at the organ level), disabilities (consequences at the level of the person) and handicaps (consequences at the level of social roles). The assessment of diagnostic data in the field of chiropody is concerned with functional problems that arise as a result of disease (for example, diabetes or rheuma) or are related to foot problems. Diagnosis in the field of chiropody is mainly at the level of impairments and less concerned with the level of disabilities and the level of handicap. Chiropody mainly aims to treat impairments related to foot problems. However, the ICIDH does not outline specific impairments related to the foot. Quite a number of impairments that occur in chiropedic practice do not therefore occur in the ICIDH. A registration form that draws on the conceptual framework of the ICIDH was developed with diagnostic categories at the impairment level which are different from the ICIDH categories.

The assessment of diagnosis using a registration form relies on clinical observations by chiropodists. It must therefore be evaluated as reliable before it can be used in a survey study. The present study is aimed at testing the 
inter-observer reliability of the assessment of diagnostic data based on clinical observations by chiropodists. One chiropodist and one trainee examined patients and level of agreement was determined, based on their observations.

\section{Method}

\section{Design}

The present study was conducted in one primary health practice, consisting of a chiropodist and a trainee. Each patient included in this study was examined by both the chiropodist and the trainee at the same time. The chiropodist and the trainee did not discuss their findings and they specified their findings independently in the registration form. The degree of agreement among their observations was determined. This procedure was also used in a study on the reliability of disability ratings by van den Berg and Lankhorst. ${ }^{4}$ For logistical reasons, it was not possible for two chiropodists to examine the patient fully independently.

\section{Registration form}

A standard registration form was used to obtain the information on patients applying for treatment." The registration form consisted of three main categories. The first category concerned general patient characteristics, complaints and the medical diagnosis by the referring physician. The second category concerned the chiropody diagnosis. The third category of the registration is concerned with treatment characteristics, but this category was not used in this study.

The International Classification of Impairments, Disabilities and Handicaps (ICIDH) was used as a framework to develop the category that was concerned with the recording of the clinical observations of the chiropodist. Two sections were developed concerning the assessment of data regarding impairments and disabilities, respectively. In order to select relevant items for the impairments section, literature on chiropody was studied. There were also two consensus meetings with chiropodists on the chiropody diagnosis, and use was made from recently formulated proposals for revision of the ICIDH, including chiropedic diagnostic categories. $^{5}$

The impairments section was subdivided into a number of categories, each consisting of several items

*Requests for reprints of the registration form (in Dutch) to Dr J. Dekker, Netherlands Institute for Primary Health Care, P.O. Box 1568, 3500 BN Utrecht, The Netherlands.
(Table 3). If the chiropodists diagnosed an impairment during the examination of the patient, it was specified in this section, together with the localization(s) of the impairment. The chiropodists could specify a maximum of four localizations (Appendix A).

The disabilities section was also subdivided into a number of categories (Appendix C). The chiropodist had to indicate the severity of the disability on a threepoint scale (Appendix B).

\section{Chiropodists}

In this study one private practice in primary health care participated, consisting of one male chiropodist and one female trainee (Table 1).

Table 1 Training and experience of the chiropodists.

\begin{tabular}{lll}
\hline & \multicolumn{2}{c}{ practice chiropody } \\
& therapist & trainee \\
\hline Year of graduation & 1987 & - \\
Experience (years) & 4 & 3rd year of study \\
Additional training & physical therapy & - \\
\hline
\end{tabular}

\section{Procedure}

The chiropodists received a brief period of training in the practical use of the registration form, in which they assessed data on five patients applying for treatment. The data on these patients were mutually compared and commented on by the researcher. These five patients were not included in this study. The chiropodists also received instructions in writing (a manual) and verbal instruction (assistance by telephone) about the use of the registration form.

All patients applying for treatment were eligible for inclusion. Patients were excluded if they had received treatment in the previous 3 months, or if they required acute treatment or refused to participate. Indications for referral were established by referring physicians, and were classified according to the International Classification of Diseases, 9 th revision, Clinical Modification (ICD-9-CM). ${ }^{6}$ The clinical details are shown in Table 2.

During the first session, information on general patient characteristics and chiropody diagnosis was assessed by both the trainee and the chiropodist at the same time. Afterwards they separately filled out the registration form. During the examination of the patient there was no exchange of information. Only if the chiropodist thought it was necessary to correct the trainee or to give the patient more information about the treatment, was information exchanged. This only occurred once, according to the chiropodists. 


\section{M.J.W.M. van Gisbergen et al.}

\section{Data analysis}

The level of agreement on impairments was determined by comparing the data concerning the observations of both chiropodists. This was done by comparing the localizations specified on each impairment. The chiropodist could specify up to a maximum of four localizations. If they both specified only one localization, these were compared in the analysis, but if they specified more than one localization, the first specified localization was used in the analysis. Impairments that are related to a specific localization (for example, respiratory problems) were analysed as present or absent.

The degree of agreement between the observers was determined using two measures, the percentage of agreement, which does not correct for agreement arising from chance, and Cohen's Kappa, ${ }^{7}$ a measure of association which does correct for chance agreement. ${ }^{8}$ In this study the percentage of agreement was designated satisfactory if the value was more than $80 \%$. In the present study the interpretation of Kappa is guided by the terminology used in the study by Fleiss ${ }^{9}$ (see also van Triet et al. ${ }^{10}$ ). Excellent agreement is indicated by a Kappa value of more than 0.75 ; agreement is satisfactory when Kappa is between 0.40 and 0.75 , and a value of less than 0.40 indicates a low level of agreement.

The frequency distribution of some impairments appeared to be skewed, in that they were identified among only a few patients. This is a problem for Kappa because a small change in skewness (especially at the extremes) can cause a large change in Kappa. ${ }^{10,11}$ There are no criteria for relating Kappa to the skewness of observations. Therefore the following criteria were chosen for interpretation; Kappa was not determined if each therapist recorded an impairment (with a certain localization) or a disability in less than $10 \%$ of patients. If an impairment or disability was recorded in 10-20\% of the patients, Kappa was calculated but has to be interpreted carefully, and if an impairment or disability was reported in more than $20 \%$ of the patients, Kappa was calculated and interpreted in a straightforward manner (van Triet et al. ${ }^{10}$ ). The percentage of agreement was determined for all impairments and disabilities.

\section{Results}

\section{Patients}

The clinical details of all patients are listed in Table 2 . The table shows that a total of 49 patients entered the study. Indications for referral were established for only
Table 2 Patient characteristics

\begin{tabular}{lr}
\hline $\begin{array}{l}\text { gender } \\
\text { male }\end{array}$ & 7 \\
female & 42 \\
medical diagnosis $(+I C D-9-C M$-codes $)$ & \\
hallux valgus $(735 \cdot 0)$ & 1 \\
hammertoe $(735 \cdot 4)$ & 1 \\
clavus $(700)$ & 1 \\
heelspur $(726 \cdot 73)$ & 1 \\
calcaneovalgus $(736 \cdot 76)$ & 1 \\
ulcus $(707 \cdot 1)$ & 1 \\
decubitus $(707)$ & 5 \\
mortonse neuralgia $(355 \cdot 6)$ & $38^{1}$ \\
other diagnosis & 49 \\
no medical diagnosis & 5 \\
total number of patients &
\end{tabular}

122 cases without a referral (and therefore no medical diagnosis) and 16 cases with a referral but no medical diagnosis.

11 patients. The indications for referral reported were related to foot problems. The percentage of female patients is higher than that of male patients.

The mean age of patients was 51 years (SD 19 years). A total of 28 patients $(57 \%)$ were referred by a general practitioner, five patients $(10 \%)$ were referred by a specialist and 16 patients $(33 \%)$ applied without referral.

\section{Chiropody diagnosis}

Impairments. In Table 3 the percentage of agreement and kappa of impairments and disabilities are listed for chiropody. Table 3 shows that in the chiropody practice only two (7\%) out of 31 impairments are reported for more than $20 \%$ of the patients. Table 3 also shows that the percentage of agreement for the 17 impairments diagnosed by the chiropodists is more than $80 \%$ for all impairments. The values vary between $94 \%$ and $100 \%$.

Table 3 shows that the Kappa values for stance deviation of foot/nail and for pain are greater than 0.75 , indicating excellent agreement. Those impairments that were observed in $10-20 \%$ of the patients have Kappa values greater than $0 \cdot 75$. A total of 14 impairments were not observed at all.

Disabilities. No meaningful analysis could be performed on the disabilities because they were observed in only two patients.

\section{Discussion}

Overall, it can be concluded that the results show that reliability of the assessment of impairments based on clinical observations, using a registration form derived from the ICIDH, is satisfactory. Kappa is excellent for 
Table 3 Inter-rater reliability of impairments

\begin{tabular}{|c|c|c|}
\hline Impairments (+ ICIDH-codes) & percentage of agreement & kappa \\
\hline \multicolumn{3}{|l|}{ Musculoskeletal impairments } \\
\hline Kyphosis $(70 \cdot 50-70 \cdot 53)$ & ** & $* *$ \\
\hline Lordosis $(70 \cdot 50-70 \cdot 53)$ & ** & ** \\
\hline Scoliosis $(70 \cdot 50-70 \cdot 53)$ & ** & $* *$ \\
\hline Other postural impairment $(70 \cdot 55,70 \cdot 58,70 \cdot 59)$ & 100 & * \\
\hline Impairment of pelvic/-torsion $(70 \cdot 70,70 \cdot 71)$ & 100 & * \\
\hline Restricted range of joint motion active $(71 \cdot 0,71 \cdot-1)$ & 100 & * \\
\hline Restricted range of joint motion passive $(71 \cdot 0,71 \cdot 1)$ & 100 & $1 \cdot 00^{1}$ \\
\hline Impairment of walking $(71 \cdot 92-71 \cdot 99)$ & 100 & * \\
\hline Impairment of coordination $\left(74 \cdot\left[4,74 \cdot \_5\right)\right.$ & ** & ** \\
\hline Hypertone muscle $\left({ }^{* * *}\right)$ & ** & ** \\
\hline Hypotone muscle $(* * *)$ & ** & ** \\
\hline Contracture $(82 \cdot 2,84 \cdot 3)$ & **. & ** \\
\hline Stance deviation of foot/toe(s) $(71 \cdot 7,71 \cdot 90,71 \cdot 91)$ & 100 & $1 \cdot 00$ \\
\hline Amputation $(75,76,79)$ & $* *$ & $* *$ \\
\hline \multicolumn{3}{|l|}{ General impairments } \\
\hline Pain $(94 \cdot 2,95 \cdot 4,95 \cdot 5,96 \cdot 3-96 \cdot 5,97 \cdot 4,97 \cdot 5,98 \cdot 3,98 \cdot 4)$ & 94 & $0 \cdot 87$ \\
\hline Fatigue $(94 \cdot 6)$ & ** & ** \\
\hline Numbness (incl. paraesthesia) $(95-97,98 \cdot 6)$ & 100 & $*$ \\
\hline Impairment of skin $(87 \cdot 0-87 \cdot 8)$ & ** & ** \\
\hline Impairment of blood circulation (***) & ** & $* *$ \\
\hline \multicolumn{3}{|l|}{ Deformities } \\
\hline Swelling $(84 \cdot 4-84 \cdot 6)$ & 100 & $*$ \\
\hline Exostosis $\left({ }^{* * *}\right)$ & 100 & * \\
\hline Heelspur $(* * *)$ & 100 & * \\
\hline Callus $(87 \cdot 8)$ & 98 & $0.88^{1}$ \\
\hline Verruca $(87 \cdot 8)$ & ** & ** \\
\hline Clavus $(87 \cdot 8)$ & 98 & $0.91^{I}$ \\
\hline Scar tissue $(87 \cdot 8)$ & $* *$ & $* *$ \\
\hline Hypertrophy of nail $(87 \cdot 9)$ & 100 & * \\
\hline Nail coming loose $(87 \cdot 9)$ & ** & ** \\
\hline Ingrowing nail $(87.9)$ & 100 & $*$ \\
\hline Hyperconvex nail $(87 \cdot 9)$ & 100 & * \\
\hline Atrophia of sole of the foot $(87 \cdot 8)$ & 100 & $1 \cdot 00^{1}$ \\
\hline
\end{tabular}

**the impairment is not observed; ${ }^{*}$ the impairment is observed in less than $10 \%$ of the patients; ${ }^{1}$ the impairment is observed in $10-20 \%$ of the patients; $\left({ }^{* * *}\right)$ no ICIDH code.

six impairments. The percentage of agreement on all reported impairments is high, although it should be noted that only 17 impairments were assessed by the chiropodists. A number of impairments were not diagnosed at all. Furthermore, it is possible that the procedure could have influenced the degree of agreement. Because both chiropodists observed patients together, their observations might also be biased.

The chiropodists participating in this study noted that some items had to be more specific, especially those concerning stance deviation of foot, nail and toe(s). They also noted that some often-appearing impairments were still missing in the impairments section and that some listed impairments were less appropriate for chiropody. Thus, it was not thought necessary to discriminate between active and passive restricted range of joint motion and low and high muscle tone. The chiropodists indicated that stance deviation of foot/toe(s) as well as impairment of nail(s) should be more specific."

*Appendix $\mathrm{C}$ shows the modified registration form.
No meaningful analysis could be performed on the disabilities because they were observed in only two patients. Apparently the disabilities played no important role in the chiropody diagnosis on the patients in this study. Disabilities may be important in other patients and for other chiropodists. However, it seems that chiropodists attend to the level of impairments in their treatment, and therefore diagnosis in the field of chiropody is mainly at this level and less concerned with the level of disabilities. In the disability section some minor modifications were made, based on comments of the participating chiropodists.*

It can be concluded that the impairment part of the ICIDH is an adequate framework for chiropody diagnosis. Although some modifications in the impairments section of the registration form appeared to be necessary, chiropodists could specify their diagnostic findings in the form. The reliability of the assessment of impairments based on clinical observations appeared to be satisfactory. 


\section{J. W.M. van Gisbergen et al.}

\section{References}

1 Dekker J, Driessen MJ, van Gisbergen M. Programmering van toekomstig onderzoek op het gebied van enkele paramedische beroepen. Utrecht: NIVEL, 1991.

2 Koster MK, Dekker J, Groenewegen PP. The Position of some Paramedical Professions in the United Kingdom, The Netherlands, the Federal Republic of Germany and Belgium. Utrecht, NIVEL, 1991.

3 World Health Organization. International Classification of Impairments, Disabilities and Handicaps. Geneva: WHO, 1980.

4 van den Berg JP, Lankhorst GJ. Inter-rater and intra-rater reliability of disability ratings based on the modified D Code of the ICIDH. International Disability Studies 1990; 12: 20-21.

5 Stichting Wetenschap en Scholing Fysiotherapie. Verslag fase 1: classificaties en definities van begrippen voor de paramedische bewegingsberoepen. Amersfoort: SWSF, 1991.

Appendix A For certain impairments, localization(s) could be indicated.

\begin{aligned} & \hline 1 Head and neck \\ & 2 Shoulder \\ & 3 Arm \\ & 4 Wrist, hand and fingers \\ & 5 Breast and abdomen \\ & 6 Spine and pelvic area \\ & 7 Hip and upper leg \\ & 8 Knee (joint) and shin/calf \\ & 9 Ankle (joint) \\ & 10 Toe(s) (joint) \\ & 11 Metatarsus \\ & 12 Mediotarsus \\ & 13 Tarsus \\ & 14 Not specified \\ & \hline\end{aligned}

6 Commission on Professional and Hospital Activities. The International Classification of Diseases, 9th Revision, Clinical Modification (ICD-9-CM). Ann Arbor, MI: CHPA, 1978.

7 Cohen J. A coefficient for agreement for nominal scales. Educational and Psychological Measurement 1960; 20: 37-46.

8 Schouten HJA. Statistical Measurement of Interobserver Agreement (dissertation). Utrecht: drukkerij Elinkwijk, 1985.

9 Fleiss L. Statistical Methods for Rates and Proportions, 2nd edn. New York: John Wiley, 1981.

10 van Triet EF, Dekker J, Kerssens JJ, Curfs EChr. Reliability of the assessment of impairments and disabilities in survey research in the field of physical therapy. International Disability Studies 1990; 12: 61-65.

11 Soeken KL, Prescott PA. Issues in the use of Kappa to estimate reliability. Medical Care 1986; 24: 733-741.

Appendix B For all disabilities the severity could be indicated on a three-point scale.

$0=$ able to perform activities on one's own

$1=$ able to perform activities, with some difficulty or with help of others

$2=$ not able to perform activities 


\section{Reliability of the diagnosis of impairments in chiropody}

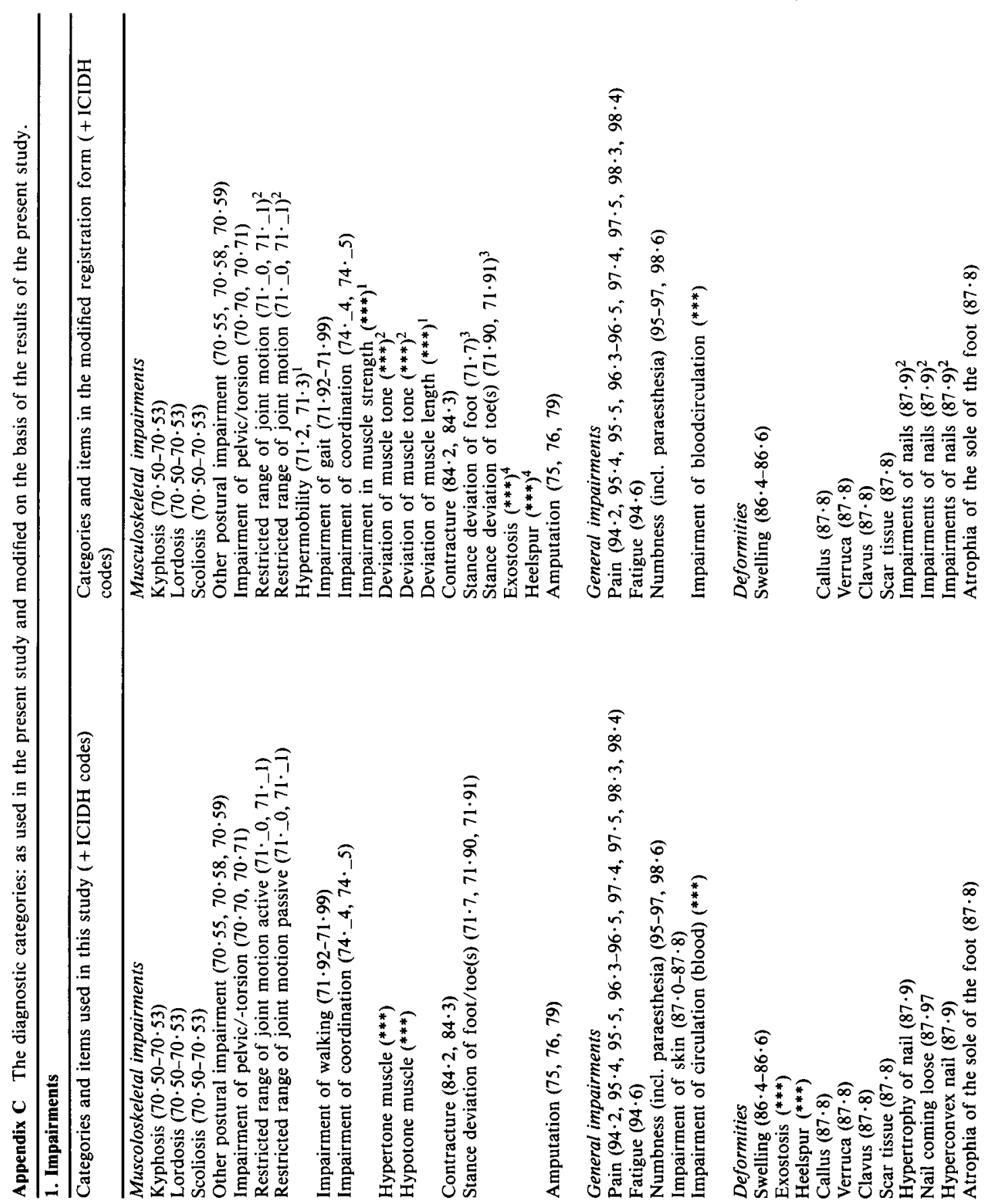


M.J.W.M. van Gisbergen et al.
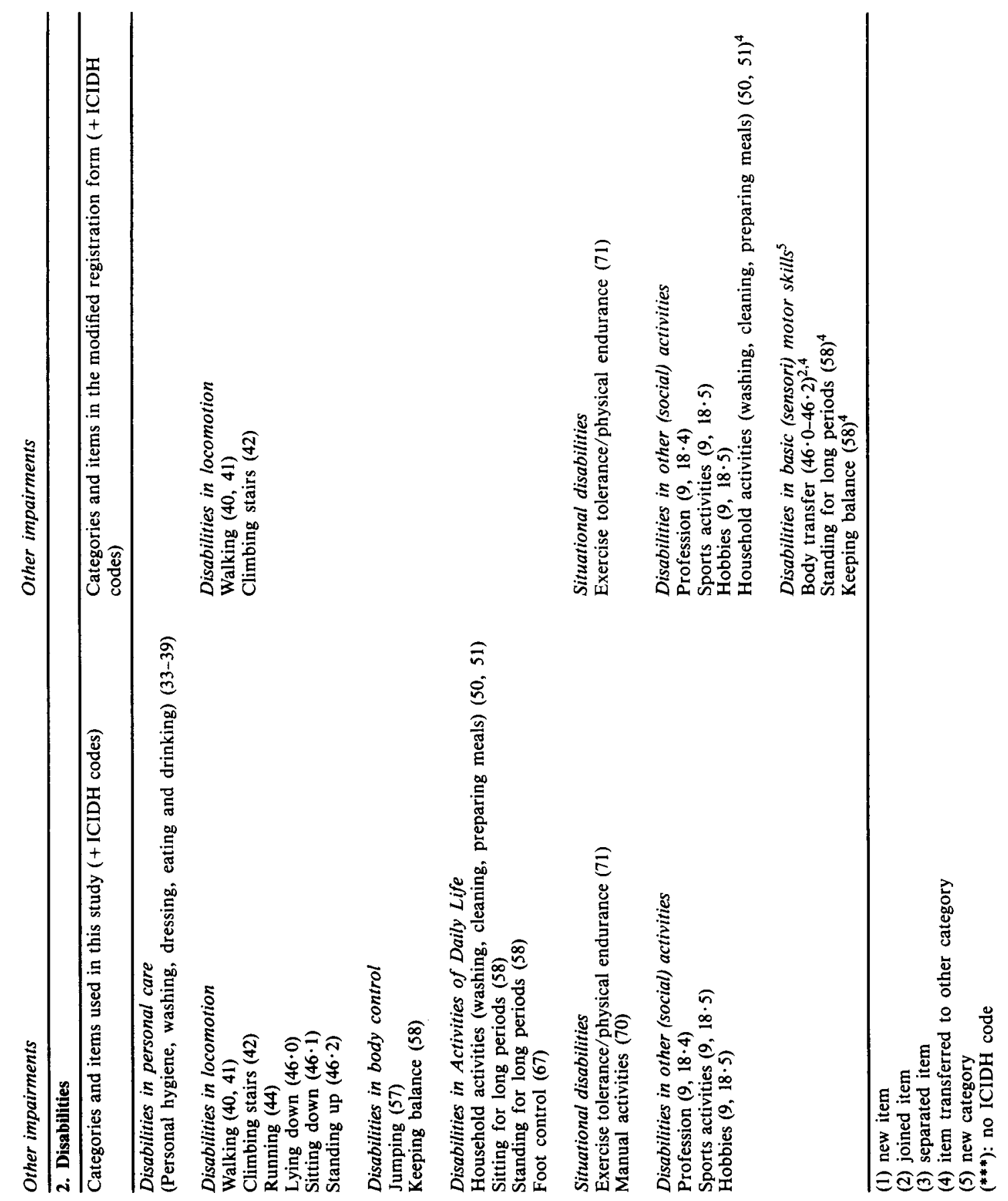